\title{
On the Quantum Baker's Map and its Unusual Traces
}

\author{
Arul Lakshminarayan \\ Physical Research Laboratory, \\ Navarangapura, Ahmedabad, 380009, India.
}

\begin{abstract}
The quantum baker's map is the quantization of a simple classically chaotic system, and has many generic features that have been studied over the last few years. While there exists a semiclassical theory of this map, a more rigorous study of the same revealed some unexpected features which indicated that correction terms of the order of $\log (\hbar)$ had to be included in the periodic orbit sum. Such singular semiclassical behaviour was also found in the simplest traces of the quantum map. In this note we study the quantum mechanics of a baker's map which is obtained by reflecting the classical map about its edges, in an effort to understand and circumvent these anomalies. This leads to a real quantum map with traces that follow the usual Gutzwiller-Tabor like semiclassical formulae. We develop the relevant semiclassical periodic orbit sum for this map which is closely related to that of the usual baker's map, with the important difference that the propagators leading to this sum have no anomalous traces.
\end{abstract}

\section{To Appear in Annals of Physics.}




\section{Introduction}

The semiclassical analysis of classically non-integrable systems has been an active field of research in the past few years. One of the central problems is the determination of the spectra of classically chaotic systems. To date the only approach to this has been via the semiclassical periodic orbit sum, which relates the traces of quantum propagators to a weighted sum over classical periodic orbits. The classical ingredients are the actions of the periodic orbits, their stabilities and their Maslov indices [20]. While this sum has been the backbone of much of the semiclassical analysis of classically chaotic systems it is known to be plagued by a lack of convergence and a loss of accuracy with increasing time. Thus the study of simple systems like certain piecewise linear maps on the torus can be very helpful in understanding some of the difficulties involved. Simple abstract maps have been quantized and studied over the years, including the well known family of continuous automorphisms, the cat maps $[9,14,15,16]$, and the piecewise linear baker's map $[9,4,5]$. Unfortunately the cat maps have a very non-generic quantum mechanics, with an exactly periodic propagator (in time), and a periodic orbit sum that is exact.

The quantum baker's map [4,5] has become a textbook example of quantizing a simple classically chaotic system [6]. The simplicity of the classical map is preserved in the quantization which has revealed many generic features, including random matrix like spectral fluctuations and eigenfunction scarring $[4,5,7]$. In the semiclassical regime a periodic orbit sum can be written for the traces of the propagator that is formally like the GutzwillerTabor periodic orbit sum $[2,3]$. However all such analysis treat the fixed points, and hence the simplest trace of the map, separately as there is no linearizable neighbourhood about such points. A more careful semiclassical analysis [1] which paid attention to the essential discreteness of the quantum map revealed that there were terms of the order of $\log (\hbar)$ in the simplest traces of the map and hence such corrections will have to be incorporated into the periodic orbit sum. These semiclassically divergent terms deserve to be further explored to see if they are generic of classically chaotic systems allowing a Markov partition or whether they are peculiar to the quantum baker's map.

The baker's map is essentially defined on a square with boundaries. Imposing periodicity, classically, makes the fixed points non-generic, in the sense that the corner fixed points at $(0,0)$ and $(1,1)$ become "half hyperbolic points" and could give rise to unusual semiclassical behaviour. Thus we would ideally like to impose some other boundary conditions while quantizing the map. Here we will adopt reflective boundary conditions on the classical map itself, thereby making it a "redundant" four bakers' map. This will create four classically disjoint baker's maps. Now this map will by its very construction have a natural topology on a torus. A quantization of this map is then possible along the lines adopted in the original quantization of the baker's map [4]. We will find that the trace of this quantum map has no semiclassically divergent terms. We also will prove that the trace of the time $T$ semiquantum propagator, obtained by quantizing the classical $T$ step map has no $\log (\hbar)$ terms, semiclassically.

In section 2 we will briefly review the classical baker's map [9,5] and discuss its 4-fold 
(classically redundant) version. In section 3 we will write down the quantum map after discussing some essential features of quantum mechanics on a torus. In section 4 we discuss the semiclassical trace of the redundant baker's map and compare it with the trace of the usual quantum baker's map. We compare the semiclassics of the semiquantum at time two for the two maps. We will also study numerically the convergence of the traces of the exact and the semiquantum propagators at time two. In section 5 we will generalize some results of section 4 and develop the semiclassical periodic orbit sum which has the canonical form of the Gutzwiller-Tabor sums. In section 6 we end with a discussion of our results.

\section{The Classical Maps}

The classical baker's map is given by :

$$
\left(q^{\prime}, p^{\prime}\right)= \begin{cases}(2 q, p / 2) & \text { if } 0 \leq q<1 / 2 \\ (2 q-1,(p+1) / 2) & \text { if } 1 / 2 \leq q<1\end{cases}
$$

$q$ and $p$ are interpreted as "position" and "momentum" in dimensionless units. The phase space is a half open square and hence toral boundary conditions may appear natural. There is one fixed point at the origin $(0,0)$. If we tessellate the plane with such open squares it is equivalent to having imposed toral boundary conditions. When we examine the behaviour of points in the neighbourhood of the fixed point we find that they do not have a generic hyperbolic structure, in that they are only a "half of a hyperbolic point". An unusual classical picture emerges if we impose periodic boundary conditions in position and momentum. This also becomes clear if we define the map on the closed square instead of the half open one. Then there are two fixed points $(0,0)$ and $(1,1)$, while the points $(0,1)$ and $(1,0)$ are not fixed. Thus we cannot treat the four corners of the classical map on a same footing as two of them are fixed points with a half neighbourhood of a normal hyperbolic point while the other two are not fixed.

The dynamics of the area preserving map eq.(1) is well known. It is completely chaotic, has a Lyapunov exponent of $\log 2$, and is a Bernoulli shift on two symbols. Further details of this map may be found in the references. Quantization of this map was first done in [4] wherein periodic boundary conditions were imposed on the states. While this is the most elegant quantization, as has been noted above, it has some unusual semiclassical features. While the quantum properties "in the bulk" are properly accounted for, there are logarithmically divergent "corner regimes" [1], creating significant departures from standard semiclassical results. Starting from the notion that these are generated by the boundary conditions of the quantum map we seek to modify the classical map itself with the least damage to its dynamics.

We first impose reflective boundary conditions on the classical baker's map. Imagine that the edges of the baker defined on the closed square are mirrors. After one reflection about the edges it is clear that the images tessellate the plane and we can impose toral boundary conditions (figure 1). We can do this for any map on the square. We consequently have four 
baker's maps that are disjoint and are on a torus. Thus the classical map is not globally ergodic, but has four distinct regions of equal area where the dynamics is chaotic.

We can scale the map so that the four bakers are on the unit square. This will be the map that we will quantize. It is clear that classically this map is just the baker's map that is made three times redundant, but quantum mechanically there are essential differences. Most importantly the four baker's maps can now be on a torus. The four corners of the unit square are fixed points and so is the center $(1 / 2,1 / 2)$. These are generic fixed points.Also while the four classical baker's are isolated, quantum mechanically they are not. There will be tunnelling amongst these classically non-interacting bakers. Such tunnelling effects in the context of other juxtaposed bakers' maps are also discussed in [10].

The extremely piecewise nature of the four bakers' map may appear to preclude quantization, as one cannot define quantum projection operators on these partitions. In fact we can view the classical map more "holistically", and this will be the key to quantization. We can write the four bakers' map as follows.

$$
\left(q^{\prime}, p^{\prime}\right)= \begin{cases}(2 q(\bmod 1),(p+[2 p]) / 2) & \text { if } 0 \leq q<1 / 4 \\ & \text { or if } 3 / 4 \leq q<1 \\ (2 q-1 / 2,(p+1 / 2) / 2) & \text { if } 1 / 4 \leq q<3 / 4 .\end{cases}
$$

Square brackets stand for the integer value function. Thus we see that in one time step the rectangle $(1 / 4,3 / 4) \times(0,1)$ gets linearly transformed into $(0,1) \times(1 / 4,3 / 4)$ (figure 1$)$. We can think of the dynamics in the other partitions similarly. First shift all the partitions $1,2,3,4$ by $(1 / 2,1 / 2)$, so that the fixed point at the corner gets shifted to the center. One can then perform the same operation as for the partitions 5,6,7,8, and finally shift the fixed point at the center back to the corner. This is exactly how we will quantize the map, as it then requires dividing the Hilbert space into two orthogonal subregions, one corresponding to the classical partitions 5,6,7,8 and the other to the partitions $1,2,3,4$, both of which trivially exist.

\section{Quantum Map}

\subsection{Preliminaries}

We will now discuss some aspects of quantum kinematics on the torus $[8,5]$ that are essential for the quantization of the four bakers' map. Imposing periodic boundary conditions on both position and momentum, results in a state space of finite dimensions. The Planck constant is related to the dimensionality of the space $N$ by

$$
2 \pi \hbar=N^{-1}
$$

The quantization in [4] was modified in [5], incorporating antiperiodic boundary conditions on the states to restore the full symmetry of the classical map, and this we will adopt. 
The position and momentum eigenvectors are given by

$$
\begin{aligned}
\left|q_{n}\right\rangle & =\left|\frac{n+1 / 2}{N}(\bmod 1)\right\rangle n=0,1, \ldots, N-1, \\
\left|p_{m}\right\rangle & =\left|\frac{m+1 / 2}{N}(\bmod 1)\right\rangle m=0,1, . ., N-1,
\end{aligned}
$$

with the transformation functions being discrete versions of plane waves:

$$
\left\langle p_{m} \mid q_{n}\right\rangle=N^{-1 / 2} e^{-2 \pi i(m+1 / 2)(n+1 / 2) / N} \equiv\left(G_{N}\right)_{m n} .
$$

$G_{N}$ is a discrete Fourier transform on $N$ sites. The symmetry operation of reflection about the center of the square $(q \rightarrow 1-q, p \rightarrow 1-p)$ is incorporated quantally by the matrix $R_{N}$ defined as

$$
\left\langle q_{n}\left|R_{N}\right| q_{n^{\prime}}\right\rangle=\delta\left(n+n^{\prime}+1, N\right)
$$

which is zero except for the secondary diagonal which has ones. The position translation operator is $V$. It is such that

$$
\left\langle q_{n}\right| V=\left\langle q_{n+1}\right|, \text { and } V^{N}=-1 .
$$

The translation operator in momentum, $U$, is similarly defined. The analogue of the uncertainty relation is the non-commutativity of $U$ and $V$ described by

$$
U V=e^{-2 \pi i / N} V U
$$

The matrix $V$ is diagonal in the momentum basis,

$$
\left\langle p_{m}|V| p_{m^{\prime}}\right\rangle=\delta_{m, m^{\prime}} e^{2 \pi i(m+1 / 2) / N}
$$

and similarly $U$ is diagonal in the position basis.

There are several useful relations amongst the matrices introduced above and all of them may be verified by direct computations. Further identities in [5] are also useful in proving some of the relations below. The act of classically translating by $(1 / 2,1 / 2)$ on the torus is an essential ingredient of the quantization. Hence we will define the unitary operator [5]

$$
T=e^{i \pi N / 4} U^{N / 2} V^{-N / 2}
$$

We note that as long as $N$ is divisible by four (which we assume throughout this paper) the operators $U^{N / 2}$ and $V^{-N / 2}$ commute, thus there is no ambiguity in defining this operator.

$T$ has the following properties;

$$
\begin{aligned}
T^{2} & =1 \\
T G_{N} & =-G_{N} T
\end{aligned}
$$

and

$$
T R_{N}=R_{N} T
$$

which may be established by using the identities given above and those in [5]. 


\subsection{Quantum dynamics}

With the preliminaries established above, one may quickly go to the heart of the quantization [4] by first quantizing the transformation of the partitions 5,6,7,8 (figure 1). In the mixed, momentum-position, representation it is given by the matrix operator which we denote as $u_{0}$, where

$$
u_{0}=\left(\begin{array}{cccc}
0 & 0 & 0 & 0 \\
0 & g_{11} & g_{12} & 0 \\
0 & g_{21} & g_{22} & 0 \\
0 & 0 & 0 & 0
\end{array}\right)
$$

and

$$
G_{N / 2} \equiv\left(\begin{array}{ll}
g_{11} & g_{12} \\
g_{21} & g_{22}
\end{array}\right) .
$$

$G_{N / 2}$ is the $N / 2$ dimensional Fourier transform that is centrally placed in $u_{0}$ which is itself an $N$ dimensional matrix. The $N / 4$ dimensional matrices $g_{i j}$ forming the larger matrix $G_{N / 2}$ are written here for future usage. The rest of the matrix elements in $u_{0}$ are zero. Thus in position representation the transformation of the partitions $5,6,7,8$ is given by the matrix $G_{N}^{-1} u_{0}$.

Following the discussion of the classical map in the previous section, we can now write the transformation of the partitions $1,2,3,4$ as

$$
T^{-1} G_{N}^{-1} u_{0} T=T G_{N}^{-1} u_{0} T,
$$

utilizing the identity $T^{2}=1$. Thus the whole quantum map, in the position representation, for the four bakers' map is given by the $N$ dimensional matrix

$$
\begin{aligned}
B & =T G_{N}^{-1} u_{0} T+G_{N}^{-1} u_{0} \\
& =G_{N}^{-1}\left(-T u_{0} T+u_{0}\right) .
\end{aligned}
$$

In the last equality we have used the anticommutation property in eq.(9). We have added the two matrices for the transformation of the partitions $1,2,3,4$ and 5,6,7,8 as the corresponding quantum subspaces in the Hilbert space are mutually orthogonal. Though unitarity is not manifest, there are certain advantages of this representation of the quantum map that we can immediately exploit.

The advertised advantages are the symmetry properties of the quantum map. The classical map has the symmetries of reflection about the center of the square and translation in phase space by $(1 / 2,1 / 2)$. The corresponding quantum symmetries are implemented by the matrices $R_{N}$ and $T$, eqs.(4,8). From the representation of the quantum map $B$ in eq.(14), it is clear that

$$
[B, T]=0, \quad\left[B, R_{N}\right]=0 .
$$

The square brackets define the usual commutator, and the above follows from the commutation of $T$ with $R_{N}$, eq.(10), and the commutation of $R_{N}$ with $u_{0}$. Thus the quantum map 
has two exact symmetries corresponding to the classical map. Since $T^{2}=1, R_{N}^{2}=1$ the eigenvalues of the operators $T$ and $R_{N}$ are \pm 1 , hence the eigenfunctions can be labelled by two good quantum numbers, and there are four symmetry classes.

There is another classical symmetry that is obvious from the way we have constructed the four bakers' map, the symmetry of reflection about the line $q=1 / 2$ (and $p=1 / 2$ ). This is translated quantally by requiring that the quantum map $B$ satisfy the relation

$$
R_{N} B^{*} R_{N}=B
$$

Here $*$ denotes complex conjugation. This symmetry is an antiunitary symmetry and is hence implemented by $R_{N} K$, where $K$ is the complex conjugation operator. When this symmetry is combined with the commutation of $B$ with $R_{N}$, eq.(15), we get the condition

$$
B^{*}=B
$$

that is $B$ is a real quantum map. Thus the unitarity of $B$ will infact establish it to be an orthogonal matrix, and the quantum four bakers' map can also be thought of as a "simple" irrational rotation in a real $N$ dimensional vector space.

Another antiunitary symmetry of the map, which it shares with the usual quantum baker's map, is the symmetry of time reversal [4]. The classical symmetry is one of interchanging position and momentum and going one step back in time. This is implemented by the operator $G_{N} K$, where $K$ is once more the conjugation operator. Thus we require the quantum map $B$ to satisfy the condition that

$$
\begin{aligned}
& G_{N} B^{*} G_{N}^{-1}=B^{-1} \text { or } \\
& G_{N} B G_{N}^{-1}=B^{t},
\end{aligned}
$$

where we have used the as yet unexplicit reality and orthogonality of $B$, and $B^{t}$ is the transpose of $B$. The cheapest way to verify the above is to take the transpose of $B$ as defined in eq.(14), and noting that while $G_{N}^{-1}$ and $u_{0}$ are symmetric, $T$ is antisymmetric. Thus the quantum four bakers' map is time reversal symmetric.

We will now write the quantum map $B$ in more explicit forms so that its reality and orthogonality will become evident. Using the definition of the translation operator $T$ in eq.(8), we can write $B$ from eq.(14) as the matrix

$$
B=G_{N}^{-1}\left(\begin{array}{cccc}
(-1)^{N / 4} g_{11} & 0 & 0 & (-1)^{N / 4} g_{12} \\
0 & g_{11} & g_{12} & 0 \\
0 & g_{21} & g_{22} & 0 \\
(-1)^{N / 4} g_{21} & 0 & 0 & (-1)^{N / 4} g_{22}
\end{array}\right)
$$

(For some more details see the Appendix). From this form one may infer the unitarity of $B$, as $G_{N}^{-1}$ is an unitary matrix, and the second matrix product's unitarity follows from that of $G_{N / 2}$. We note here some features of this matrix. Firstly there is a Planck constant dependent phase in front of one block of the Fourier transform that corresponds to the 
classical transform of the partitions $1,2,3,4$. This phase factor is simply \pm 1 as we have assumed $N$ to be divisible by four. But for this phase factor, $T$ symmetry would be exact only if $N$ is divisible by 8 . Secondly this matrix form might have been written by inspection using the methods outlined in [4]. While this would have missed out the phase factor, we note that such phase factors sitting globally on blocks do not affect the classical limit. They might affect such properties as traces, and symmetries.

Further evaluation of matrix elements is possible, using the basic definitions of the components of $B$ as Fourier transforms. After performing a few elementary geometric sums, we get the following explicit real representation of $B$ in the position basis, $\left\langle q_{n^{\prime}}|B| q_{n}\right\rangle$, as

$$
\begin{aligned}
& (-1)^{N / 4} \frac{\sqrt{2}}{N} \frac{\sin \left[\pi\left(n^{\prime}-2 n-1 / 2\right) / 2\right]}{\sin \left[\pi\left(n^{\prime}-2 n-1 / 2\right) / N\right]} \quad \text { if } \quad 0 \leq n \leq N / 4-1, \\
& \frac{\sqrt{2}}{N} \frac{-\sin \left[\pi\left(n^{\prime}+1 / 2\right) / 2\right]}{\cos \left[\pi\left(n^{\prime}-2 n-1 / 2\right) / N\right]} \quad \text { if } \quad N / 4 \leq n \leq 3 N / 4-1, \\
& (-1)^{N / 4} \frac{\sqrt{2}}{N} \frac{-\sin \left[\pi\left(n^{\prime}-2 n-1 / 2\right) / 2\right]}{\sin \left[\pi\left(n^{\prime}-2 n-1 / 2\right) / N\right]} \quad \text { if } \quad 3 N / 4 \leq n \leq N-1 .
\end{aligned}
$$

Due to the time reversal symmetry, the momentum space representation is also real, and infact just the transpose of $B$. The matrix elements of the quantum map are thus simple elementary functions, a feature that it shares with the single baker's map. The classical limit of $B$ corresponds to the four fold redundant baker, and this occurs in the same way as for the single baker's map. From the classical map it may be expected that the quantum map be nearly block diagonal (with blocks of size $N / 2$ ) in both position and momentum basis. This is infact the case, and moreover the "tunnelling" matrix elements are not exponentially small in Planck constant, but rather go as $1 / N$ as may be inferred from eq.(20). This leads to rather large tunnelling effects as time goes by.

\subsection{Properties of the Quantum Map}

The quantum four bakers' map is real and preserves all classical symmetries. It is therefore of interest to study the spectral properties of this map, and compare it with those of the quantum baker's map. As this takes us away from the central thrust of this article we will merely state some results, although a much more comprehensive study is definitely desirable. The symmetry group underlying the quantum map is isomorphic to the Abelian dihedral group $D_{2}$ consisting of four elements $(I, R, T, R T)$. Here $I$ is the identity matrix. The symmetry $R T$, the combined symmetry of reflection about the center of the square and translation in phase space by $1 / 2$ is the " $R$ symmetry" of the usual baker's map, which corresponds to the symmetry of reflection about the center of each individual baker's map. It is satisfying that even this symmetry is present in the quantum map. The existence of the discrete commuting canonical symmetries of $R$ and $T$ makes the labelling of states by two quantum numbers possible. In particular the eigenstates belong to one of the four possible classes, denoted as $|++\rangle,|+-\rangle,|-+\rangle,|--\rangle$. All the eigenangles (in units of $2 \pi$ ) are irrational, a property it shares with the quantum baker's map. 
There are no degeneracies in the quantum map. However the reality of the map implies that eigenvalues occur as complex conjugate pairs, which belong to different irreducible representations of the quantum map (with different $T$ eigenvalues), and hence to different symmetry classes. The reduced four bakers' map is block diagonal with four blocks. The following unitary matrix simultaneously diagonalizes both $R$ and $T$, and hence can be used in the reduction of the quantum map. If we write

$$
\begin{gathered}
P= \\
\frac{1}{2}\left(\begin{array}{cccc}
1 & 1 & 1 & 1 \\
-i R_{N / 4} A_{N / 4} & i R_{N / 4} A_{N / 4} & i R_{N / 4} A_{N / 4} & -i R_{N / 4} A_{N / 4} \\
-i A_{N / 4} & i A_{N / 4} & -i A_{N / 4} & i A_{N / 4} \\
R_{N / 4} & R_{N / 4} & -R_{N / 4} & -R_{N / 4}
\end{array}\right),
\end{gathered}
$$

then $P^{-1} B P$ is a block diagonal matrix with four blocks. Where

$$
\left(A_{N / 4}\right)_{m n}=(-1)^{m} \delta_{m n}, \quad m, n=0, \ldots, N / 4-1 .
$$

One may exploit these symmetries to reduce the size of matrices that need diagonalization. For example all $|++\rangle$ eigenvalues can be got by diagonalising the $N / 4$ dimensional matrix

$$
B_{11}-i B_{12} R_{N / 4} A_{N / 4}-i B_{13} A_{N / 4}+B_{14} R_{N / 4},
$$

where $B_{m n}$ is the $m n^{\text {th }}$ block of the quantum map divided into sixteen $N / 4$ dimensional matrices.

\section{The Semiclassical Traces}

\subsection{The Anomalous Traces}

In this subsection we recall some features of the quantum baker's map and its unusual traces [1]. Consider the quantum baker's map, denoted here as $B^{\prime}$ and defined as

$$
B^{\prime}=G_{N}^{-1}\left(\begin{array}{cc}
G_{N / 2} & 0 \\
0 & G_{N / 2}
\end{array}\right)
$$

The simplest trace in the quantum problem is $\operatorname{Tr}\left(B^{\prime}\right)$, and in [1], we find its semiclassical (large N) form,

$$
\operatorname{Tr} B^{\prime} \sim 2 \sqrt{2}\left[\frac{1}{4}-\frac{i}{2 \pi}(\log (8 N / \pi)+\gamma)\right]
$$

where $\gamma$ is the Euler constant.

An aspect of the quantum baker's map that has been exploited in its semiclassical analysis is the possibility of quantizing the map after a certain number of classical iterations. Thus

for instance if we quantize the baker's map after two time steps $\left(B^{(2) \prime}\right)$, we would not get the 
same operator as $B^{\prime 2}$. The differences between the two families of operators (quantum and semiquantum, in the terminology of ref. [1]) is of great interest, as it reflects the differences between quantal and classical evolutions. In [1] we find a careful semiclassical comparison of the matrix elements of the above two operators, and beautiful visual representations of the same.

Part of the differences may be captured in the trace metric [11], as we can derive the following semiclassical result;

$$
\operatorname{Tr}\left(B^{\prime 2} B^{(2) \prime \dagger}\right) \sim N-\frac{4}{\pi^{2}} \log (N)+\alpha
$$

where $\alpha=\frac{4}{\pi^{2}}\left(\log \left(\frac{\pi}{8}\right)+4 \beta(2)-\gamma-1\right) \approx 0.46686 \ldots \beta(2)$ is Catalan's constant [12]. The singular semiclassical term in both of the above traces is logarithmic. The trace of the propagator is governed semiclassically by the fixed points of the map, and the logarithmic behaviour in the first trace is a reflection of the corner configuration of this fixed point. Eq.(24) measures the "distance" between the two operator families, the quantum and the semiquantum in the Euclidean norm, and it seems to indicate semiclassical divergence (in the norm) even if only marginally.

The trace of the propagator at time two is itself difficult to estimate. While we can do a semiclassical evaluation of the trace of $B^{(2) \prime}$, the same for $B^{\prime 2}$ is not available, except for some partial results in [1]. In ref. [1] the semiclassical form of $\operatorname{Tr}\left(B^{(2) \prime}\right)$ is decomposed in accordance with classical symbol sequences. This exact symbolic decomposition of the propagator at both the quantum and the semiquantum level is an interesting feature of the baker's map, and is true only at the semiquantum level for the four bakers' map. Let the quantum propagator associated with the transformation of the partition 00 be $b_{00}^{\prime}$. Partition 00 is the first of the four equal vertical partitions of the phase space square. For complete definitions see ref. [1].

For our immediate purposes it is sufficient to note that the trace of $b_{00}^{\prime}$ is governed semiclassically by the fixed point $(0,0)$. Similarly the transformation of the partition 10 is given by the propagator $b_{10}^{\prime}$, whose trace is semiclassically governed by the fixed point $(1 / 3,2 / 3)$. Note that for the time two map, there are four classical fixed points, and that the propagators $b_{00,10}^{\prime}$ are not unitary. The complete trace of $B^{(2) \prime}$ is given by $2 \operatorname{Tr}\left(b_{00}^{\prime}+b_{10}^{\prime}\right)$. This is a consequence of $R$ symmetry [1]. Again in ref. [1] we find the semiclassical form of these traces which we repeat here for comparison with the corresponding four bakers' traces.

$$
\begin{gathered}
\operatorname{Tr}\left(b_{00}^{\prime}\right) \sim \frac{-i}{3 \pi} \log N+\frac{2}{3}\left\{\frac{1}{4}-\frac{i}{2 \pi}\left[\log \frac{8}{3 \pi}+2 \log (1+\sqrt{2})+\gamma\right]\right\}, \\
\operatorname{Tr}\left(b_{10}^{\prime}\right) \sim \frac{2}{3} e^{4 \pi i N / 3}
\end{gathered}
$$

We note that once again there are terms logarithmic in the Planck constant in the semiquantum trace governed by the corner fixed point. There are no such terms for the trace of $b_{10}^{\prime}$ which is controlled by the "good" fixed point in the interior. 


\subsection{Four Bakers' Semiclassical Traces}

We now evaluate the semiclassical trace of the four bakers' map, whose real position basis representation is given by eq.(20). Following ref. [1] we can do a careful aymptotic analysis, in order to avoid possible pitfalls, but in fact elaborate analysis is not necessary and we will see, as can be expected, that it is legal here to replace summations with integrals. We will write the traces in forms that explicitly resemble those of the usual quantum baker's map. From the original definition of $B$ in eq.(19), we will define the following non-unitary matrices.

$$
\begin{aligned}
& b_{0}=e^{i \pi N / 4} G_{N}^{-1}\left(\begin{array}{cccc}
g_{11} & 0 & 0 & 0 \\
0 & 0 & 0 & 0 \\
0 & 0 & 0 & 0 \\
0 & 0 & 0 & 0
\end{array}\right) \\
& \bar{b}_{0}=e^{i \pi N / 4} G_{N}^{-1}\left(\begin{array}{cccc}
0 & 0 & 0 & 0 \\
0 & 0 & 0 & 0 \\
0 & 0 & 0 & 0 \\
g_{21} & 0 & 0 & 0
\end{array}\right)
\end{aligned}
$$

Then we get

$$
\operatorname{Tr} b_{0}=e^{i \pi N / 4} \frac{\sqrt{2}}{N} \sum_{m=0}^{N / 4-1} \sum_{n=0}^{N / 4-1} e^{-2 \pi i(m+1 / 2)(n+1 / 2) / N}
$$

and

$$
\operatorname{Tr} \bar{b}_{0}=e^{i \pi N / 4} \frac{\sqrt{2}}{N} \sum_{m=0}^{N / 4-1} \sum_{n=0}^{N / 4-1} e^{2 \pi i(m+1 / 2)(n+1 / 2) / N} .
$$

These further imply that

$$
\begin{gathered}
\operatorname{Tr}\left(b_{0}+\bar{b}_{0}\right)=e^{i \pi N / 4} \frac{2 \sqrt{2}}{N} \operatorname{Re}\left(\sum_{m=0}^{N / 4-1} \sum_{n=0}^{N / 4-1} e^{-2 \pi i(m+1 / 2)(n+1 / 2) / N}\right) \\
=e^{i \pi N / 4} \frac{2 \sqrt{2}}{N} \cdot \frac{1}{4} \sum_{m=-N / 4}^{N / 4-1} \sum_{n=-N / 4}^{N / 4-1} e^{-2 \pi i(m+1 / 2)(n+1 / 2)} ;
\end{gathered}
$$

we get the final form by reflecting the summation lattice about $m=0$ and $n=0$. This form belongs to the "regular regime", that is there are no corners in the summation and asymptotically it can be approximated by a continuum integral, followed by a stationary phase approximation. We then extend the range of integration and evaluate the integral exactly, as it is a Gaussian.

Thus we get

$$
\operatorname{Tr}\left(b_{0}+\bar{b}_{0}\right) \sim e^{i \pi N / 4} \frac{\sqrt{2}}{2 N} \int_{-\infty}^{\infty} d p \int_{-\infty}^{\infty} d p e^{-2 \pi i N p q}=e^{i \pi N / 4} \frac{\sqrt{2}}{2} .
$$


Finally using both $R$ and $R T$ symmetries we get

$$
\operatorname{Tr} B=4 \operatorname{Tr}\left(b_{0}+\bar{b}_{0}\right) \sim e^{i \pi N / 4} 2 \sqrt{2} .
$$

Thus we see the close relationship between the real part of the quantum baker's trace, eq.(23), and the trace of the four bakers' map, and it comes as no surprise that semiclassically the regular part of the trace of the baker's map is quarter that of the four bakers' map's trace. We will observe in the following that this is true also beyond time one.

The absolute value of the asymptotic trace is what one will expect with the semiclassical Gutzwiller-Tabor periodic orbit sum, although we have not attempted to develop the semiclassics of the map yet (we will do so in section 5). We can also compare the above form, eq.(31), with the analogous sum of the usual quantum baker's map. We have from [1]

$$
\operatorname{Tr} B^{\prime}=\frac{2 \sqrt{2}}{N} \sum_{m=0}^{N / 2-1} \sum_{n=0}^{N / 2-1} e^{-2 \pi i(m+1 / 2)(n+1 / 2) / N} .
$$

Admittedly, while the four bakers' map promises more "generic" behaviour than the baker's map, beyond the first trace we have not proved that such is the case. However some qualitative arguments may be put forward towards this end. Beyond time one, a very comprehensive semiclassical analysis for the baker's map has been attempted in [1] for time two. They found for instance that not only were the time two quantum and semiquantum traces significantly different for the corner regime that is controlled by the fixed point at the corner, but so also were the "bulk traces" governed by the "good" period two points.

A qualitative argument put forward to explain this [1] is the presence of aliasing, when there are "quasi-stationary" points in the saddle point approximation. At time two, such points converge on the origin which anyway has a singular semiclassical behaviour, leading to discrepancies even in the "bulk". In the quantum four bakers' map, since the corner regime is not singular, we may expect such aliasing effects to create only "benign" behaviours. Thus the semiclassical four baker does indeed promise to be more "generic".

\subsection{The Time Two Semiquantum Map}

As has been noted earlier, a key ingredient in the semiclassical quantum baker's map has been the semiquantum propagators. At time two this is constructed out of the quantization of the time two classical map. Indeed the simplicity of the classical baker's map and the versatality of the quantization procedure makes this possible. The author is not aware of any other map where the construction of such (non-trivial) semiquantum propagators exists. This at once makes the quantum baker's map special as well as valuable. The construction of semiquantum propagators is also possible for the four bakers' map.

Here however we will adopt the quantization scheme in [4] neglecting phase factors at blocks and then getting the phase factors by imposing $T$ symmetry. It has been mentioned earlier that this is possible at the level of the time one map itself. For arguments concerning 
the general case see the Appendix. We can write the semiquantum map for time two as the matrix

$$
G_{N}^{-1}\left(\begin{array}{cccccccc}
e^{i \pi N / 8} g_{11} & 0 & 0 & 0 & 0 & 0 & 0 & e^{i \pi N / 8} g_{12} \\
0 & 0 & g_{11} & 0 & 0 & g_{12} & 0 & 0 \\
0 & g_{11} & 0 & 0 & 0 & 0 & g_{12} & 0 \\
0 & 0 & 0 & g_{11} & g_{12} & 0 & 0 & 0 \\
0 & 0 & 0 & g_{21} & g_{22} & 0 & 0 & 0 \\
0 & g_{21} & 0 & 0 & 0 & 0 & g_{22} & 0 \\
0 & 0 & g_{21} & 0 & 0 & g_{22} & 0 & 0 \\
e^{i \pi N / 8} g_{21} & 0 & 0 & 0 & 0 & 0 & 0 & e^{i \pi N / 8} g_{22}
\end{array}\right),
$$

where

$$
G_{N / 4} \equiv\left(\begin{array}{ll}
g_{11} & g_{12} \\
g_{21} & g_{22}
\end{array}\right) .
$$

We have used a generic symbol $g_{i j}$ to denote the blocks for both time one and two. It must be emphasized that these are not the same. We just do not want to clutter up the symbols with the dimensionality of the blocks, and we hope that this does not create any undue confusion. Here we have assumed that $N$ is divisible by eight. One may note that the structure of the matrices is a reflection of the structures of the quantum baker's map [1]. The Fourier blocks are at classical period two points of the four bakers' map. The preservation of classical symmetries makes $B^{(2)}$ a real quantum map too.

In figure 2 we show the ratios $\operatorname{Tr}\left(B^{2}\right) / \operatorname{Tr}\left(B^{(2)}\right), \operatorname{Tr}\left(B^{\prime 2}\right) / \operatorname{Tr}\left(B^{(2) \prime}\right)$, corresponding to the quantum four bakers' map and the quantum baker's map. In the last ratio the plot is split into the ratio of the real part of the traces and the imaginary part of the traces. In the first ratio the operators are themselves real, and so will be the traces. The bold line in the figure represents the case of the four bakers' map, while the dashed lines correspond to the baker's map. The convergence in the case of the four bakers' map of the traces of the quantum and the semiquantum propagators is apparent. This is to be compared with the behaviour of the quantum baker's map, where the deviations of the ratios from unity are quite large. This is in spite of the fact that at the same value of the Planck constant, the effective Planck constant for a single baker of the four bakers' map is four times larger. Thus a more comprehensive study of the quantitative differences between the semiquantum and quantum propagators of the four bakers' map is warranted.

\subsection{Semiclassics of Semiquantum at Time Two}

We can provide a semiclassical analysis of the time two semiquantum propagator with relative ease, and we do so here. We wish to once more decompose the dynamics according to classical symbol sequences. There are four independent bakers and hence we can write the dynamics as a full shift on four independent binary symbols. We will concentrate on two of them as the other two will be related to these by $R$ symmetry. Explicitly we will denote 
by $b_{00}$ the matrix

$$
e^{i \pi N / 8} G_{N}^{-1}\left(\delta_{11} \otimes g_{11}\right)
$$

Here $\delta_{i j}, i, j=1, \ldots 8$ are eight dimensional matrices with a one at the $\mathrm{i}^{\text {th }}$ row and the $\mathrm{j}^{\text {th }}$ column, the rest being zero. They serve to indicate the positioning of the $N / 8$ dimensional blocks in the mixed representation of $B^{(2)}$. Compare with eq.(35). The above equation tells us to select only the upper left most block in the mixed representation. The trace of this operator would be semiclassically governed by the fixed point $(0,0)$. we write similarly

$$
\begin{gathered}
\bar{b}_{00}=e^{i \pi N / 8} G_{N}^{-1}\left(\delta_{81} \otimes g_{21}\right) \\
b_{10}=G_{N}^{-1}\left(\delta_{32} \otimes g_{11}\right) \\
\bar{b}_{10}=G_{N}^{-1}\left(\delta_{62} \otimes g_{21}\right)
\end{gathered}
$$

The overbars are only to indicate the blocks that are classically reflections of each other. As for time one we may now invoke both $R$ and $R T$ symmetries to write the complete trace as

$$
\operatorname{Tr} B^{(2)}=4 \operatorname{Tr}\left(b_{00}+\bar{b}_{00}+b_{10}+\bar{b}_{10}\right)
$$

An explicit calculation gives us

$$
\begin{aligned}
& \operatorname{Tr}\left(b_{00}+\bar{b}_{00}\right)= \\
& e^{i \pi N / 8} \frac{2}{N} \sum_{m=0}^{N / 8-1} \sum_{n=0}^{N / 8-1} e^{-6 \pi i(m+1 / 2)(n+1 / 2) / N}\left(1+e^{3 \pi i(m+1 / 2) / 4}\right) \\
& =e^{i \pi N / 8} \frac{4}{N} \operatorname{Re}\left[\sum_{m=0}^{N / 8-1} \sum_{n=0}^{N / 8-1}\left(e^{-6 \pi i(m+1 / 4)(n+1 / 2) / N}\right)\right] \\
& =e^{i \pi N / 8} \frac{2}{N} \sum_{m=0}^{N / 8-1} \frac{\sin (3 \pi(m+1 / 2) / 4)}{\sin (3 \pi(m+1 / 2) / N)}
\end{aligned}
$$

The corresponding double sum that led to eq.(25) for the usual baker's map is [1]

$$
\frac{2}{N} \sum_{m=0}^{N / 4-1} \sum_{n=0}^{N / 4-1} e^{-6 \pi i(m+1 / 2)(n+1 / 2) / N} .
$$

The regular behaviour of the real part of the trace of the usual quantum baker's map was already noted in [1], and shown that in fact the real part was in the "regular regime". The four bakers' map picks out as relevant only the real part of the trace, eq.(43), thus it should not come as a surprise if there are no singular traces in the model. This is also expected since there are no corners on the torus defining the phase space of the four bakers' map. Thus there are no "half hyperbolic points". However the deeper reasons, if any, for the regular behaviour of the real part is not known to the author, and the four bakers' map may provide an interesting model to shed some light on this question as it chooses only the regular real part. 
As was done for time one, in eq.(43) reflect the summation lattice about $m=0$ and $n=0$, to obtain the following.

$$
\operatorname{Tr}\left(b_{00}+\bar{b}_{00}\right)=e^{i \pi N / 8} \frac{1}{N} \sum_{m=-N / 8}^{N / 8-1} \sum_{n=-N / 8}^{N / 8} e^{-6 \pi i(m+1 / 2)(n+1 / 2) / N}
$$

which is a sum in the regular regime, there are no corners in the summation, and it is again legal to replace the sum asymptotically by an unbounded continuum approximation, resulting in

$$
\operatorname{Tr}\left(b_{00}+\bar{b}_{00}\right) \sim e^{i \pi N / 8} N \int_{-\infty}^{\infty} d p \int_{-\infty}^{\infty} d q e^{-6 \pi i N p q}=\frac{1}{3} e^{i \pi N / 8}
$$

More elaborate, "brute force" [1] methods of evaluating the semiclassical trace confirm the above result, thus we are justified in adopting conventional wisdom in this model.

We can write the equations for the "regular regime" by similar explicit evaluations. In particular we write,

$$
\operatorname{Tr} b_{10}=\frac{2}{N} e^{i \pi N / 3} \sum_{m=N / 8}^{N / 4-1} \sum_{n=N / 4}^{3 N / 8-1} e^{-6 \pi i(n+1 / 2-N / 3)(m+1 / 2-N / 6) / N}
$$

and

$$
\operatorname{Tr} \bar{b}_{10}=\frac{2}{N} e^{-i \pi N / 3} \sum_{m=N / 8}^{N / 4-1} \sum_{n=5 N / 8}^{3 N / 4-1} e^{-6 \pi i(n+1 / 2-2 N / 3)(m+1 / 2-N / 6) / N}
$$

In eq.(49) when we reflect the $n$ summation by means of the transform $n \rightarrow N-n-1$, we get the exact conjugate of $\operatorname{Tr} b_{10}$. Thus we get, after applying the unbounded continuum approximation,

$$
\begin{gathered}
\operatorname{Tr}\left(b_{10}+\bar{b}_{10}\right) \sim 4 N \operatorname{Re}\left[e^{i \pi N / 3} \int_{-\infty}^{\infty} d p \int_{-\infty}^{\infty} d q e^{-6 \pi i N(q-1 / 3)(p-1 / 6)}\right] \\
=\frac{4}{3} \cos \left(\frac{\pi N}{3}\right)
\end{gathered}
$$

and thus finally the complete trace of $B^{(2)}$ can be written from eq.(41) as

$$
\operatorname{Tr} B^{(2)} \sim e^{i \pi N / 8} \frac{4}{3}+\frac{16}{3} \cos \left(\frac{\pi N}{3}\right) .
$$

Comparing these results with the corresponding formulae for the usual quantum baker's map eqs. $(25,26)$ we see that once more the four baker traces are semiclassically four times the regular part when 16 divides $N$. We note here, without proof, that while the traces of the quantum and the semiquantum propagators had just enough cancellations to remove terms of order $\log (\hbar)$, the Euclidean norm marginally diverges whether the classical map has corners (usual baker's map) or not (four bakers' map). Further work on this is needed as the results are inconclusive. 


\section{$5 \quad$ Periodic Orbit Sum}

Following the results of the previous section it does not require great intuition to understand the semiclassical periodic orbit sum of the four bakers' map. In this section we extend the semiclassical analysis of the semiquantum maps upto times where it is defined, and thereby arrive at a periodic orbit sum for the quantum four bakers' map. The existence of such a sum for the usual quantum baker's map is well known [1,3], but the four bakers' map has the added advantage that there are no anomalous terms and we do not have to treat the fixed points specially. The symbol $T$ has been used in other sections of this article to denote translational symmetry, but in this section it will denote time.

We have to first describe the time $T$ semiquantum propagator. We will concentrate first on the upper left half of the propagator in the mixed momentum-position representation. Thus the blocks populating this area will be $N / 2^{T+1}$ dimensional matrices which we will write generically as $g_{11}$. The construction of this propagator requires that $2^{T+1} \mid N$; we will assume a slightly stronger condition that $2^{T+2} \mid N$. This assumption will do away with any phases that globally sit on blocks (i.e., they become one). The semiquantum propagator is defined only upto time $T+1$, and the expressions we write down will be valid only upto time $T$. We locate the $g_{11}$ blocks, like the blocks are located for the usual quantum baker's map, at the classical periodic orbits. The upper left hand corner of the matrix corresponds to the origin of the classical phase space.

Let $\nu_{L}$ be a binary string of length $L$ and its binary evaluated value be $\nu$. We denote by $\bar{\nu}$ the bit reversed, binary evaluated integer of the same string. For example, if $L=2$ and $\nu_{L}=01$, then $\overline{\nu_{L}}=10, \nu=1$ and $\bar{\nu}=2 .(\nu, \bar{\nu})$ uniquely determines a classical periodic orbit of length $L$ and a $g_{11}$ block in the semiquantum propagator. The classical periodic orbit is located $[1,3]$ at

$$
q_{0}=\frac{\nu}{2\left(2^{L}-1\right)}, \quad p_{0}=\frac{\bar{\nu}}{2\left(2^{L}-1\right)} .
$$

This refers to the periodic orbits in the lower left, principal baker's map. It differs from the location of the periodic orbits in the usual baker's map by a trivial scaling factor of $1 / 2$. The semiquantum propagator block that is controlled by this periodic orbit is a matrix of dimension $N / 2^{T+1} \times N / 2^{T+1}$ and is given in the mixed representation by

$$
\sqrt{\frac{2^{T}}{N}} \exp \left[-\frac{2^{T+1} \pi i}{N}\left(m+\frac{1}{2}-\frac{\nu N}{2^{T+1}}\right)\left(n+\frac{1}{2}-\frac{\bar{\nu} N}{2^{T+1}}\right)\right]
$$

where

$$
\begin{aligned}
m & =\nu N / 2^{T+1}, \ldots,(\nu+1) N / 2^{T+1}-1, \\
n & =\bar{\nu} N / 2^{T+1}, \ldots,(\bar{\nu}+1) N / 2^{T+1}-1 .
\end{aligned}
$$

For convenience we define

$$
M \equiv N / 2^{T+1} \text {. }
$$

The "conjugate block" corresponds to the classical fixed point at $\left(q_{0}, 1-p_{0}\right)$, and exists in the upper right half baker of the classical phase space. The semiquantum blocks generically 
denoted as $g_{21}$, controlled by such periodic points, populate the lower left half of the propagator in the mixed representation. If the reader is confused, she is advised to turn to the special cases of time one and two in the previous section for comparisons. This block is once more of linear dimension $N / 2^{T+1}$, and is given by

$$
\sqrt{\frac{2^{T}}{N}} \exp \left[-\frac{2^{T+1} \pi i}{N}\left(m+\frac{1}{2}-\frac{\left(2^{T+1}-\nu-1\right) N}{2^{T+1}}\right)\left(n+\frac{1}{2}-\frac{\bar{\nu} N}{2^{T+1}}\right)\right]
$$

where

$$
\begin{gathered}
m=\left(2^{T+1}-\nu-1\right) N / 2^{T+1}, \ldots,\left(2^{T+1}-\nu\right) N / 2^{T+1}-1, \\
n=\bar{\nu} N / 2^{T+1}, \ldots,(\bar{\nu}+1) N / 2^{T+1}-1 .
\end{gathered}
$$

After a Fourier transform to the pure position representation we trace the correponding operators to get

$$
\begin{aligned}
\operatorname{Tr} b_{\nu}= & \frac{\sqrt{2^{T}}}{N} \sum_{n=\nu M}^{(\nu+1) M-1} \sum_{m=\bar{\nu} M}^{(\bar{\nu}+1) M-1} \exp \left[\frac{2 \pi i}{N}\left(m+\frac{1}{2}\right)\left(n+\frac{1}{2}\right)\right] . \\
& \exp \left[-\frac{2^{T+1} \pi i}{N}\left(n+\frac{1}{2}-\frac{\nu N}{2^{T+1}}\right)\left(m+\frac{1}{2}-\frac{\bar{\nu} N}{2^{T+1}}\right)\right] .
\end{aligned}
$$

and

$$
\begin{aligned}
\operatorname{Tr} \overline{b_{\nu}} & =\frac{\sqrt{2^{T}}}{N} \sum_{n=\left(2^{T+1}-\nu-1\right) M}^{\left.2^{T+1}-\nu\right) M-1} \sum_{m=\bar{\nu} M}^{(\bar{\nu}+1) M-1} \exp \left[\frac{2 \pi i}{N}\left(m+\frac{1}{2}\right)\left(n+\frac{1}{2}\right)\right] . \\
& \exp \left[-\frac{2^{T+1} \pi i}{N}\left(n+\frac{1}{2}-\frac{\left(2^{T+1}-\nu-2\right) N}{2^{T+1}}\right)\left(m+\frac{1}{2}-\frac{\bar{\nu} N}{2^{T+1}}\right)\right] .
\end{aligned}
$$

We can write the sum in eq.(56) as

$$
\begin{gathered}
\operatorname{Tr} b_{\nu}=\frac{\sqrt{2^{T}}}{N} \exp \left(\frac{i \pi N \nu \bar{\nu}}{2\left(2^{T}-1\right)}\right) \cdot \sum_{n=\nu M}^{(\nu+1) M-1} \sum_{m=\bar{\nu} M}^{(\bar{\nu}+1) M-1} . \\
\exp \left[-\frac{2 \pi i}{N}\left(2^{T}-1\right)\left(n+\frac{1}{2}-\frac{\nu N}{2\left(2^{T}-1\right)}\right)\left(m+\frac{1}{2}-\frac{\bar{\nu} N}{2\left(2^{T}-1\right)}\right)\right] .
\end{gathered}
$$

Similarly from eq.(57) we get

$$
\begin{gathered}
\operatorname{Tr} \bar{b}_{\nu}=\frac{\sqrt{2^{T}}}{N} \exp \left(-\frac{i \pi N \nu \bar{\nu}}{2\left(2^{T}-1\right)}\right) \cdot \sum_{n=\left(2^{T+1}-\nu-1\right) M}^{\left(2^{T+1}-\nu\right) M-1} \sum_{m=\bar{\nu} M}^{(\bar{\nu}+1) M-1} . \\
\exp \left[-\frac{2 \pi i}{N}\left(2^{T}-1\right)\left(n+\frac{1}{2}+\frac{\nu N}{2\left(2^{T}-1\right)}-N\right)\left(m+\frac{1}{2}-\frac{\bar{\nu} N}{2\left(2^{T}-1\right)}\right)\right] .
\end{gathered}
$$


In the last equation if we reflect the $n$ summation about the center, $(n \rightarrow N-n-1)$, we get the complex conjugate of the other trace, i.e.,

$$
\operatorname{Tr} b_{\nu}=\left(\operatorname{Tr} \bar{b}_{\nu}\right)^{*}
$$

where the $*$ indicates complex conjugation. Thus we now have

$$
\operatorname{Tr}\left(b_{\nu}+\bar{b}_{\nu}\right)=2 \operatorname{Re}\left(\operatorname{Tr}\left(b_{\nu}\right)\right)
$$

which is in the regular regime irrespective of whether the fixed point is at the corner or not (see the discussions in the previous section). However at the corner, that is when $\nu$ is a pure string of 1 or 0 , there is an additional step of reflecting the summation lattice about $m=0$ and $n=0$ before we will be able to apply the unbounded continuum approximation. This step leads to a factor of a quarter, which we will account for below. With this mind, we can replace the summations in eq.(58) by integrals and do a stationary phase approximation. We note that the relevant periodic point where the phase is stationary is $\left(q_{0}, p_{0}\right)$, and is given by eq. (53).

Thus we get $\operatorname{Tr}\left(b_{\nu}+\bar{b}_{\nu}\right) \sim$

$$
\begin{gathered}
2 \sqrt{2^{T}} N \operatorname{Re}\left[\exp \left(\frac{i \pi \nu \bar{\nu} N}{2\left(2^{T}-1\right)}\right) \int_{-\infty}^{\infty} d q d p \exp \left(-2 \pi i N\left(2^{T}-1\right)\left(q-q_{0}\right)\left(p-p_{0}\right)\right)\right] \\
=\frac{\cos \left(\frac{\pi \nu \bar{\nu} N}{2\left(2^{T}-1\right)}\right)}{\sinh \left(\frac{T}{2} \log 2\right)} .
\end{gathered}
$$

The complete trace of the semiquantum operator at time $T$ is, due to $R$ symmetry,

$$
\operatorname{Tr} B^{(T)}=2 \sum_{L(\nu)=T} \operatorname{Tr}\left(b_{\nu}+\bar{b}_{\nu}\right)
$$

The summation extends over all possible binary strings of length $T$.

The fixed points at the center $(1 / 2,1 / 2)$ and corner $(0,0)$ of the classical phase space have to be treated with more care, as has been stated above. The extra factor of one quarter for these points should hardly come as a surprise, as a reflection about the lines $q=1 / 2$, $p=1 / 2$, do not produce any new periodic points, unlike the "bulk" periodic orbits which create three more copies of themselves. In other words while the fixed points at $(1 / 2,1 / 2)$ and the four corners belong to all four of the bakers, the other periodic points belong to individual bakers and thus there are four copies of each. This can be accounted for by calculating the fixed point contributions explicitly. Thus we write the final semiclassics of the four bakers' map which we identify with the semiclassics of the semiquantum map at time $T$ as,

$$
\operatorname{Tr} B^{T} \sim \operatorname{Tr} B^{(T)} \sim \frac{1}{\sinh \left(\frac{T}{2} \log 2\right)}\left(1+2 \sum_{L(\nu)=T}^{\prime} \cos \left(2 \pi N S_{\nu}\right)\right) .
$$


Here $S_{\nu}=\frac{\nu \bar{\nu}}{4\left(2^{T}-1\right)}$ is the action for the classical periodic orbit. It differs by a trivial factor of a quarter from the action for the usual baker's map [3]. The prime indicates that the sum is taken over all binary strings of length $T$ except the ones which have exclusively 1 or exclusively 0 in them. The contributions from such orbits have been already explicitly added. The formulae of the previous section are special cases of this formula, for $T=1,2$.

This formula is then in the form of a Gutzwiller-Tabor periodic orbit sum, which expresses the trace of the propagator as an approximate sum over classical periodic orbits. It is essentially the same as the real part of the periodic orbit sum for the usual quantum baker's map, with the important modification that there will be no anomalous terms in the trace of the exact propagator. In figure 3 we compare the periodic orbit sum with the exact quantum traces in the time domain. Note that the short periodic orbit traces are very good approximations, unlike in the case of the usual baker's map, where the effect of the fixed points non-generic character produced large errors.

The energy domain is simply a Fourier transform away, and the reliability of eigenvalues estimated depends crucially on the time domain input. The value of $N$, the inverse of the Planck constant is 1024. We see that the periodic orbit sum tracks the exact trace for a substantially long time, as compared to the log time for each individual baker's map. Although the periodic orbit sum was derived under the assumption that $2^{T+2} \mid N$, we can evaluate the sums beyond such times. We have compared the traces for twenty time steps, and we find the continuation of tracking. While this long time semiclassical accuracy has been noted earlier [2], the individual periodic orbit traces can sometimes be a very bad estimate of the exact trace. It might be that the error is weakly bounded in time. We get a similar behaviour in another quantum map on the torus, the sawtooth map [19]. Thus the initial hope that the quantum baker's map will represent generic behaviour of quantum non-integrable systems is at least partially restored.

Recently there have been results that seem to improve the accuracy of the periodic orbit formula, either by accounting for orbits that are just about to be "born" out of a bifurcation [17] or by calculating higher order corrections [18]. It would thus be of interest to understand the possible ways in which the periodic orbit sum for the baker's map can be improved. We believe that with the removal of terms logarithmic in the Planck constant from the semiclassical formulae the way has been cleared for more such detailed studies.

\section{Discussions}

The rigorous dissection of the quantum baker's map in ref. [1], showed up many nongeneric features of the map, and the principal questions that grew out of it was whether these properties are stable against changing quantizations and models. We have demonstrated here that such features are not stable against modifications of quantization. We showed in particular that the quantum bakers as social animals tend to have normal semiclassical features. While there still remains the question of why the boundary conditions mattered so much in the quantum baker's map, it is clear that the generator of such discrepancies lies 
in the unusual configuration of the fixed point, whose trace contribution was not that of a regular hyperbolic point.

The semiclassics of the quantum four bakers' map follows a periodic orbit sum and there are no anomalous terms in the traces of the propagators generating this sum. Thus it is an ideal testing ground for the periodic orbit sum, and its validity in the time and energy domains. It has been observed that the traces of the baker's map are valid much beyond the log time $[1,2]$. While the propagators that were used to generate these traces, the semiquantum propagators, themselves do not exist, evaluating the periodic orbit sum seems to produce reasonably accurate traces. We can expect that the quantum four baker's map will follow similar behaviour, as it is essentially very similar to the usual baker's map. In fact we have seen that not only classically is the four bakers' map redundant, but also semiclassically the information from a single baker's map, the fundamental domain, was sufficient. The genericity of the results reported here depend upon the investigation of other quantum maps with chaotic classical limits. The semiclassical theory of the quantum sawtooth map [19] shows similar features.

The four bakers' map may prove to be interesting for other reasons, though it has now lost some simplicity that the baker's map had. We intend to pursue its study elsewhere. The novel feature of the four bakers' map is the tunnelling that occurs between the classically isolated bakers. Such tunnelling was found to be abnormally large in another juxtaposed baker map [11]. The tunnelling occurs between regions in phase space which have chaotic dynamics within them and are separated by a separatrix. Coherent tunnelling effects, which we have not discussed above, have been observed between diagonally opposite bakers. It is not clear at this stage how relevant such tunnelling effects are for generic dynamical systems. An exact symbolic decomposition of the quantum propagator exists for the quantum baker's map [1], based on the possible classical transitions at any given time. The possibility of tunnelling between the regions of the four bakers' map makes such a symbolic decomposition more tedious, in particular there will be classically forbidden symbol sequences in the decomposition of the exact quantum propagator.

\section{Acknowledgements}

It is a pleasure to thank Professor N.L. Balazs and Dr. A. Voros for many discussions and constant encouragement. I am grateful for the hospitality of the Service de Physique Theorique, Saclay, where the principal ideas of this note became clear. It is also a pleasure to thank Professor V.B. Sheorey for several discussions. 


\section{References}

1. M. Saraceno, and A. Voros, Physica 79 D (1994), 206.

2. P.W.O' Connor, S. Tomsovic, and E.J. Heller, Physica 55D (1992), 340.

3. A.M.O. De Almeida, and M. Saraceno, Ann. Phys., (N.Y.) 210 (1991), 1.

4. N.L. Balazs, and A. Voros, Ann. Phys., (N.Y.)190 (1989), 1.

5. M. Saraceno, Ann. Phys., (N.Y.) 199 (1990), 37.

6. L.E. ReIchl, "The Transition to Chaos in Conservative Classical Systems: Quantum Manifestations", Springer Verlag, New York, 1990.

7. P.W.O' Connor, and S.Tomsovic, Ann. Phys., (N.Y.)207 (1991), 218.

8. J. Schwinger, "Quantum Kinematics and Dynamics", Benjamin, New York, 1970.

9. V.I. Arnold, And A. Avez, "Ergodic Problems of Classical Mechanics" WA Benjamin, New York (1968).

10. A. Lakshminarayan, and N. L. Balazs, J. Stat. Phys. 77 (1994) 311.

11. A. Lakshminarayan, Doctoral Dissertation, Dept. of Physics, S.U.N.Y at Stony Brook (N.Y., 1993).

12. M.Abramowitz, AND I.A.Stegun, "Handbook of mathematical Functions ,", U.S. National Bureau of standards, Washington. D.C., 1964.

13. I.C. Percival, and F. Vivaldi, Physica 30D (1988), 164.

14. J.H. Hannay, and M.V. Berry, Physica 1D (1980), 267.

15. J. P. Keating, Nonlinearity 4 (1991), 335.

16. J. Ford, G. Mantica, And G.H. Ristow, Physica 50D (1991), 493.

17. M. Kus, F. Haake, and D. Delande, Phys. Rev. Lett. 71 (1993), 2167.

18. P. Gaspard, and D. Alonso, Phys. Rev. A47 (1993), R3468.

19. A. Lakshminarayan, Phys. Lett. 192 A (1994), 345.

20. M.C. Gutzwiller, "Chaos in Classical and Quantum Mechanics", Springer (New York, 1990).

21. M.A. Sepulveda, S.Tomsovic And E.J. Heller, Phys. Rev. Lett. 69 (1992), 402. 


\section{Figure Captions}

Figure 1. The four bakers' map before and after a transformation. Shown are the principal partitions of the map. The fundamental baker comprises of the partitions 1 and 5 . The rest are obtained by reflections.

Figure 2. Comparison of the traces of the quantum and the semiquantum operators at time two for the baker's and the four bakers' maps. A represents the case of the four bakers' map, which is a real map. $\mathrm{B}$ and $\mathrm{C}$ represent the ratio of the real and imaginary parts of the traces for the case of the usual baker's map.

Figure 3. Comparison of the periodic orbit sum traces with the exact traces, for the case when $N=1024$. The first solid line in the "doublets" represents the periodic orbit sum, while the artificially shifted lines represent the quantum traces. 


\section{Appendix}

The original formulation of the quantum four bakers' map was in the form of eq.(14), while much of the later results depend upon the matrix form of eq.(19). We derive here the latter from the former.

$$
B=G_{N}^{-1}\left(-T u_{0} T+u_{0}\right)
$$

where

$$
T=e^{i \pi N / 4} t_{1} t_{2}
$$

with

$$
t_{1}=U^{N / 2}, \text { and } t_{2}=V^{-N / 2} .
$$

$U$ and $V$ are translation operators, in momentum and position, on the torus and have been defined in eqs. $(5,6)$. We note that $t_{1}$ and $t_{2}$ commute because we have assumed $N$ to be divisible by 4 . The position representation of $V^{-N / 2}$ is given by a matrix which we conveniently write as

$$
t_{2}=\left(\begin{array}{cccc}
0 & 0 & -I_{N / 4} & 0 \\
0 & 0 & 0 & -I_{N / 4} \\
I_{N / 4} & 0 & 0 & 0 \\
0 & I_{N / 4} & 0 & 0
\end{array}\right),
$$

where $I_{N / 4}$ are $N / 4$ dimensional identity matrices. Then using the matrix representation of $u_{0},(11)$, it is easy to see that

$$
u_{1} \equiv t_{2} u_{0} t_{2}=\left(\begin{array}{cccc}
-g_{22} & 0 & 0 & g_{21} \\
0 & 0 & 0 & 0 \\
0 & 0 & 0 & 0 \\
g_{12} & 0 & 0 & -g_{11}
\end{array}\right) .
$$

Now we separate out diagonal $t_{1}$ into four $N / 4$ dimensional diagonal blocks which we write as $e^{i \pi / 2} a_{j}$, with $j=1, \ldots, 4 . \quad\left(a_{1}\right)_{m n}=(-1)^{m} \delta_{m n}, m, n=0, \ldots, N / 4-1$ and $\left(a_{j}\right)_{m n}=$ $(-1)^{N(j-1) / 4} a_{1}$, where $j=1,2,3,4$. We then get

$$
\begin{aligned}
& -T u_{0} T+u_{0}=-t_{2} u_{1} t_{2}+u_{0}= \\
& \left(\begin{array}{cccc}
a_{1} g_{22} a_{1} & 0 & 0 & -a_{1} g_{21} a_{4} \\
0 & g_{11} & g_{12} & 0 \\
0 & g_{21} & g_{22} & 0 \\
-a_{4} g_{12} a_{1} & 0 & 0 & a_{4} g_{11} a_{4}
\end{array}\right)
\end{aligned}
$$

Now a simple explicit calculation yields

$$
\begin{aligned}
& a_{1} g_{22} a_{1}=e^{i \pi N / 4} g_{11},-a_{1} g_{21} a_{4}=e^{i \pi N / 4} g_{12}, \\
& -a_{4} g_{12} a_{1}=e^{i \pi N / 4} g_{21}, a_{4} g_{11} a_{4}=e^{i \pi N / 4} g_{22},
\end{aligned}
$$

which establishes the matrix form in (19). A simple extension of the arguments here also give us the matrix form of the semiquantum propagator at time two, eq.(35), besides showing us that if $2^{T+2} \mid N$ then there are no additional phase factors. This has been have used in section 5 where we have not included any global phase factors. 